\title{
Modelling Failure Conditions in Zigbee based Wireless Sensor Networks
}

\author{
Apoorva Singh, Itu Snigdh
}

Department of Computer Science, Birla Institute of Technology, Mesra,835215, India

\begin{abstract}
Reliable data transmissions in sensor networks are important for its longevity and require research attention. Since, fault tolerance and reliability become vital for embedded systems, estimating failure scenarios aid WSN applications to establish robust backup mechanisms in case of failures in the network. The article aims to model failure in IEEE 802.15.4 Zigbee networks in order to estimate the longevity of the network in context to the network connectivity. Ideally, IEEE 802.15.4 can manage two types of networks, the star topology or the peerto-peer topology. On combining these two we get a mesh network. Hence, the article presents the failure conditions for both star and mesh based network topology to the point where the network is disconnected owing to varied failure conditions.
\end{abstract}

Index Terms: Wireless sensor networks, Fault trees, Reliability, Star, Mesh topology, Zigbee Network.

(C) 2017 Published by MECS Publisher. Selection and/or peer review under responsibility of the Research Association of Modern Education and Computer Science

\section{Introduction}

A WSN typically consists of few hundreds of smart and low-cost multimodal microsensor devices, communicating through wireless links and essentially requiring the internet for varied applications. We usually employ a WSN for coalescing the diverse possibilities of either monitoring or controlling complicated environmental conditions; for applications that may relate to disaster prevention, prediction, intrusion detection, safety and other critical concerns. This concept however essentially requires the communication among the smart sensors to be reliable and dependable. The concept of multi-hop mesh based wireless networks have been used in several wireless networking technologies, like Personal Area Networks (PANs), local area networks (LANs) and metropolitan area networks (MANs) [1].

A wireless sensor network is characterised by energy constricts, intermittent mobility, localization problems [2]. For the simplicity of analysis, we assume a sensor network that has a fixed topology based message

* Corresponding author. Tel.: +91 7033646425
E-mail address: singh.apoorva04@ hotmail.com 
transmission system. Among these networks, one of the most popular technologies is context to wireless sensor networks is based on IEEE standards 802.11s and 802.15.4 [3-6]. The article suggests reliability estimation for Zigbee networks for both peer to peer (tree based) and star (graph based) WSN architectures. Since there is an inherent conflict between power consumption and reliability; wherein an increase in reliability usually leads to an increase in power consumption we choose IEEE 802.15.4 standard for our analysis. This is simply because Zigbee provides a standard for ultra-low complexity, ultra-low cost, ultra-low power consumption and low data rate wireless connectivity among inexpensive devices [6].

Section II presents a brief discussion on previous work related to reliability aspect of Wireless Sensor Networks. Section III describes various parameters and other information related to the system modelling in Zigbee based WSNs. Section IV and section V elaborates on modelling of failure conditions in Mesh Network and Star Network based Zigbee architectures. Section VI sums up the whole workin the form of a conclusion.

\section{Related Work}

Methods to measure the reliability of the large-scale wireless sensor networks are a fundamental requirement of wireless sensor networks deployment. Reliability has been studied for multimodal fusion sensor networks [7] wherein the system reliability for the case of two and three types of sensors (modelling pooled spares) has been calculated.

Most models consider that WSN can fail in two points: links and sensor nodes [8-10]. It has been observed that the reliability of a particular region is affected by the routing protocol adopted, by the number of nodes belonging to the region and by the distance of these regions to the sink node. [11]. Also, the application communication in wireless sensor networks (WSN) depends on two important factors: acquisition of sensed data from a specific area, and network connectivity [12].

The two key QoS requirements of WSN are; Sensing coverage and Network connectivity while network lifetime based on energy efficiency and invulnerability based on fault tolerance are two main factors to measure network reliability. Reliability metrics have been developed for WSN under five different data delivery models, including sink unicast, anycast, multicast, manycast, and broadcast taking up an example of WSN with tree topology [13].

Literary works address and illustrate the effect of few scenario metrics, viz., network size, transmission range, and network coverage area, on the reliability measures (i.e., 2TRm and ATRm) by modelling MANET as Geometric Random Graph[14].Also, reliability has been discussed using a generalised binary decision diagram (BDD)-based approach is presented for evaluating the reliability of a 1-out-of-(n+1) warm standby system subject to fault level coverage[15]. A Similar approach integrates a progressive reduction scheme based on binary decision diagrams for the reliability analysis and a divide-and-conquer approach for considering the effect of CCF [16]. Reliability of WSN for different topologies, routing protocols and metrics using fault trees have been proposed [17-18]. However, none of the above research is dedicated to Zigbee topologies. Hence, our article specifically analyses the impact of the failure of Zigbee network elements with the help of fault trees.

The paper discusses the estimation of reliability with the help of fault trees. The system model outlines the network while the reliability is estimated for the star and mesh networks in section 4 and 5, followed by the conclusion.

\section{System Model}

The Zigbee network has two types of devices; FFD (a fully functional device) and an RFD (Reduced functional device). The first FFD that is activated may establish its own network and become a Personal Area Network (PAN) coordinator. Then both FFD and RFD devices can connect to the PAN coordinators that are within the radio sphere of influence of the PAN coordinator. We assume an analogous network containing three types of nodes; namely sensor nodes, relay nodes that connect directly to the sink in case of the tree network. Likewise, a mesh network has the same setup with the coordinator node as an additional node that connects to 
the sink directly and maintains the network. Reliability of the overall structure depends on the following network elements:

- Link- Links connect two member nodes or a member node to a relay node, for communication to take place

- Member nodes- Member nodes are the participating and communicating devices in the wireless sensor network

- Relay node - Relay node is analogous to the cluster head. It is responsible for relaying messages of other member nodes to the sink node, other than sending its own messages.

- Coordinator node- Coordinator nodes are assisting devices which help the relay node in managing the member nodes, in cases of dense network deployment and mesh topologies.

If reliability $(\mathrm{R}(\mathrm{t})$ ) of a network may be defined as the probability that the communication backbone (event occurrence is successfully communicated to the sink via the available routes) fails in the interval from time 0 to time $t$; availability is the parameter that is ensured when a route break or congestion occurs for most of the routing algorithms. Availability is enhanced by parallel processing, load balancing, fault tolerance, redundant coverage and efficient communication paths [2]. As a result, these network requirements are usually addressed in conjunction with the reliability of the network.

We assume network system states that are distinct due to the type of network failure they encounter. Some of the states do not disrupt message communication or network connectivity while other failure states are critical and cause failure of the network as a whole. We assume four network states in case of the mesh network while there are three significant states in case of a star network. The states' notation and their significance are presented in Table 1.

Table 1. Symbol Table

\begin{tabular}{lll}
\hline NODE ID & Symbolical Name & Network States \\
\hline $\mathbf{S}_{\mathbf{0}}$ & $\mathrm{P}$ & All nodes are working \\
$\mathbf{S}_{1}$ & $\mathrm{Z}$ & Link failure state \\
$\mathbf{S}_{2}$ & $\mathrm{Y}$ & Relay node failure state \\
$\mathbf{S}_{3}$ & $\mathrm{Q}$ & Member node failure state \\
$\mathbf{S}_{4}$ & $\mathrm{X}$ & Coordinator node failure state \\
$\mathbf{S}_{\mathrm{N}}$ & $\mathrm{R}$ & All failed states \\
\hline
\end{tabular}

\section{Mesh Network}

The Mesh topology is a dynamic kind of network, it employs redundancy and redeployment wherever possible. The possibilities considered to model failure conditions through fault tree are subject to the following failures:

1. Failure of the connecting link between the member node and the relay node. This link fails when either the connected member nodes or the connecting relay node fails.

2. Failure of the relay node or failure of connecting links due to environmental conditions. If the relay node fails the entire network fails.

3. Failure of the member nodes except the relay node. 
4. Failure of the coordinator node or failure of connecting links between the member nodes and the coordinator node. Coordinator nodes are also member nodes but with the additional responsibility of assisting the relay node when the network is vast.

\section{Case 1: Network state when link fails}

$\mathrm{S}_{1}$ denotes the states where the link fails while other network elements like the relay node member and coordinate node are active. The system failure is given by the fault tree model by fig 1 if ' $S_{0} . S_{I}\left(S_{2}+S_{4}\right)$ ' is 1 . Hence, $S_{0}, S_{1}, S_{2}, S_{4}$ are the network elements incurring subsequent failures. It can be observed that if $S_{1}$ fails one or many of the links connecting member node to relay node or member node to coordinate node or relay node to sink node are failing to lead to failure in communication to the sink.

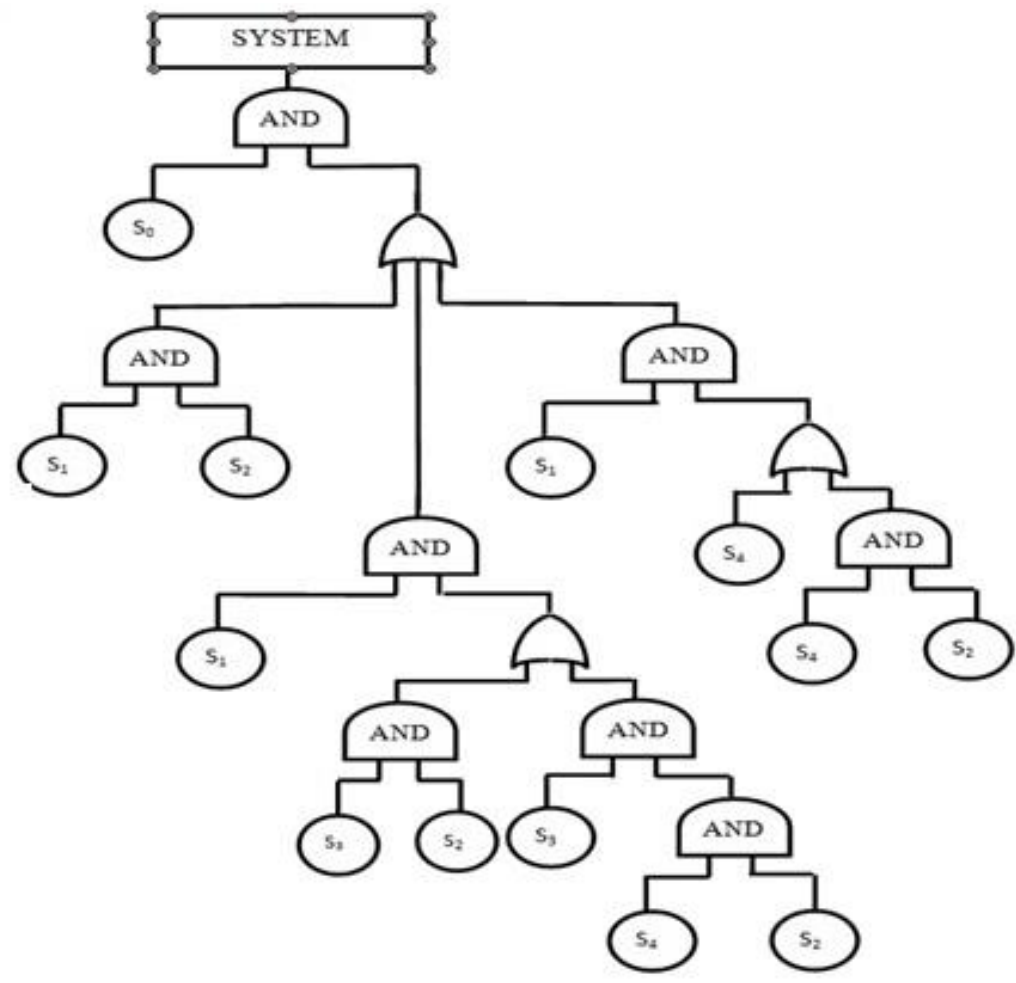

Fig.1. Fault tree when link fails

The above given fig. 1.depicts that $S_{1}$ being one of the input edges of all AND sub-trees, hence it is bound to fail even if one of the input edges are 0 .

Therefore, the fault tree simplification can be given by the equation (1), the symbolical name used is depicted in Table 1 and the reliability of the network is estimated as equation (2).

$$
R=P(Z Y(1+Q)+Z X)=P Z(Y+X)
$$

$$
R E L I A B I L I T Y=1-P Z(Y+X)
$$




\section{Case 2: Network State when Relay Node fails}

With the failure of $S_{2}$ that is the relay node failure, the whole network fails as the transfer of messages to the sink node is done through the relay node, which is modelled in figure2.The other network elements like the link, member nodes, and coordinator nodes are active.

The failure is attributed to the failure of state $\mathrm{S}_{0}, \mathrm{~S}_{1}$ asgiven by ' $\left(S_{0} . S_{2}\right)$ ' or we can say that system fails if P $\cdot \mathrm{Y}$ is 1.If $S_{2}$ fails, i.e the relay node fails then even if other network elements are active, no form of communication is possible, that can be seen in fig. 2, if $S_{2}$ state fails, an AND function would result in 0 and subsequently the system fails.

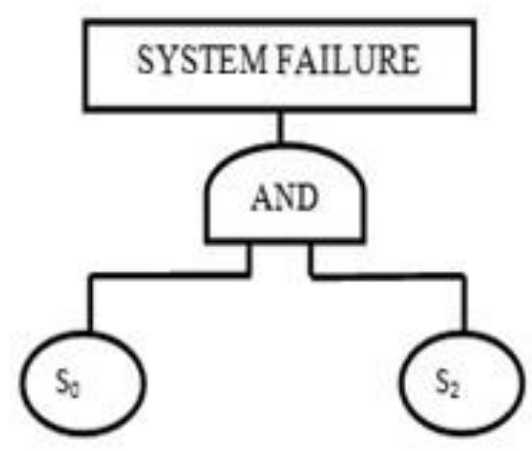

Fig.2. Fault tree when Relay node fails

The corresponding fault tree can be solved to determine the failed state of the network as a whole given by equation (3) and the corresponding reliability that can be achieved by equation (4).

$$
R=P Y
$$

$$
\text { RELIABILITY }=1-P Y
$$

Case 3: Network State when Member nodes fail

We discuss the case when the member nodes fail while the relay node, link and coordinator nodes are active. The system failure is given by ' $S_{0} \cdot S_{3}\left(S_{2}+S_{4}\right)$ ' or simply if $P Q(Y+X)$ is 1 . If $S_{3}$ fails, the member nodes cease to communicate with the relay node, even though the one or more of the other member nodes may still be communicating to the sink node through the relay node.

The fault tree given in fig. 3 is simplified as given in equation (5) where equation (6) gives the overall reliability. The given equation (5) depicts that in the case of member node failure even if the member nodes have failed, the network does not cease to exist because more than one member nodes maybe active.

$$
R=P(Q Y+Q X)=P Q(Y+X)
$$

$$
\text { RELIABILITY }=1-P Q(Y+X)
$$




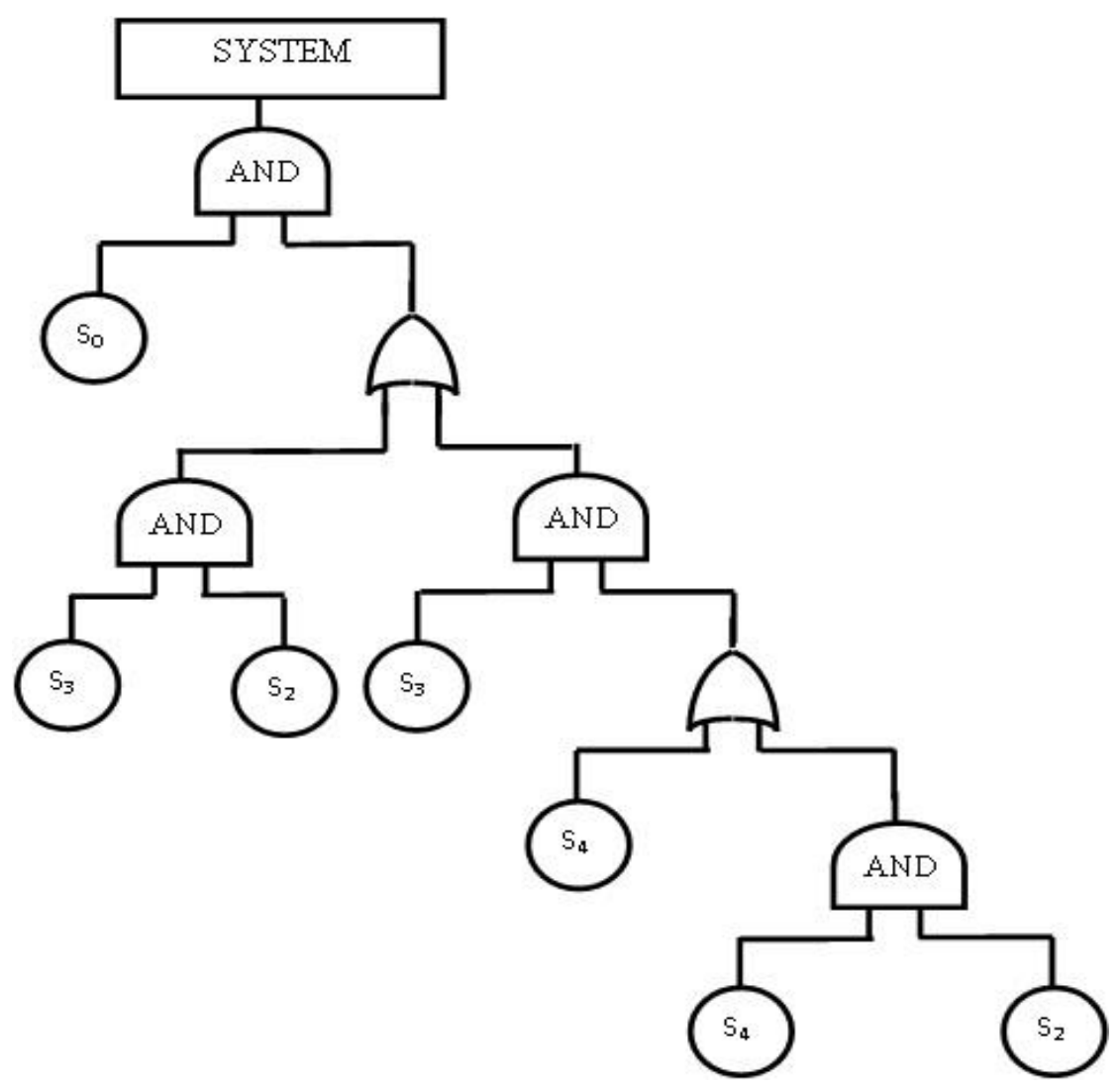

Fig.3. Fault tree when Member nodes fail

\section{Case 4: Network State when Co-ordinator node fails}

If $\mathrm{S}_{4}$ the Coordinate node is failing whereas the connected relay node is active, Link is active, Member nodes are active. Then, the system fails if $\{P \cdot X\}$ is 1 or when the output of the corresponding fault tree is ' $S_{0} \cdot S_{4}$ '. If $\mathrm{S}_{4}$ fails, then part of the network which was connected to the coordinator would cease to communicate with the relay node, even though some other parts may still be communicating as shown in fig. 4.

The system failure is given by equation (7) and the reliability by equation (8). In the case of coordinator node failure, some other part of the network may be still active and transferring the messages, and so the equation (7) depicts only the instance when the coordinator node part has failed and others are active.

$$
R=P X(1+Y)=P X
$$

RELIABILITY $=1-P X$ 


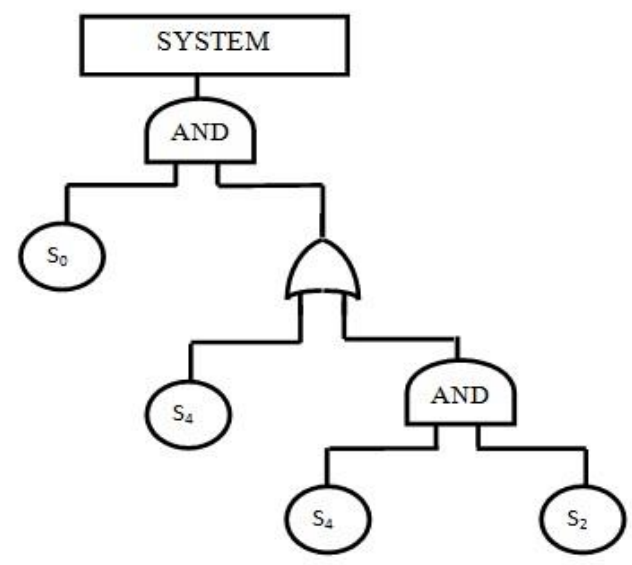

Fig.4. Fault tree when Co-ordinator node fails

\section{Star Network}

The star network topology is another very widely used topology in potential Zigbee networks. Hence we extend our analysis of reliability to such type of networks. The inherent characteristic of a star network is the central control or the single point of failure. Hence reliability of such systems is solely dependent on how we delay the failure of the main communicating node. Also, as a fact, there are not much redundant transmissions possible due to similar reasons.

Similar to the mesh network we assume that one network element fails at a time while the others are working.

This assumption gives us three possible states, as explained in the above section IV and the corresponding fault trees are depicted in Figure 5,6 and 7.The corresponding fault trees are solved to estimate the reliability for each case.

Case 1: Network State when link has failed

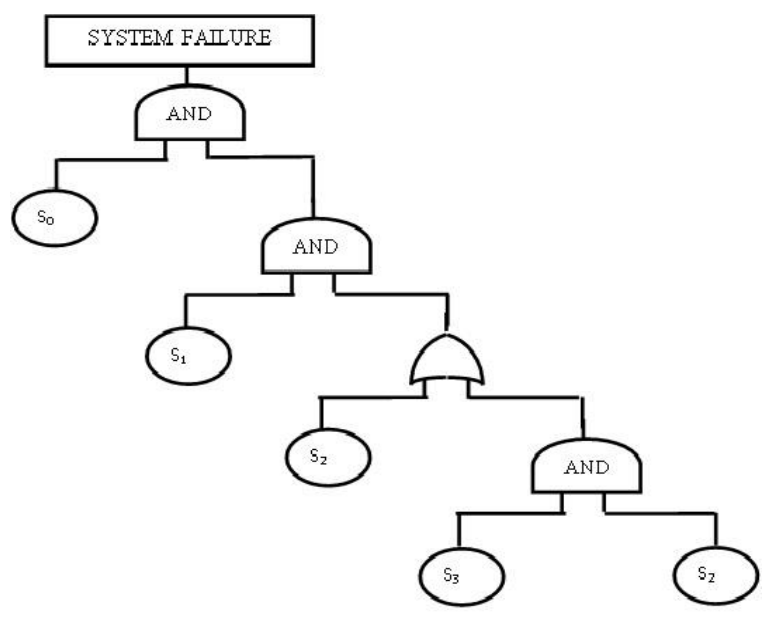

Fig.5. Fault tree when link fails 
The system fails if $\{P Z Y\}$ is 1 or when the output of the corresponding fault tree is ' $S_{0 .} S_{l} . S_{2}$ '. The fault tree depicted in fig. 5 is used to estimate the failure and reliability as depicted in equations (9) and (10).

$$
R=P Z(Y+Q Y)=P Z Y
$$

$$
\text { RELIABILITY }=1-P Z Y
$$

Case 2: Network State when relay node has failed

The corresponding fault tree denotes the relay node failure case and computes the reliability as depicted in equation (11) and (12). The system fails if $\{P Y\}$ is 1 or when the output of the corresponding fault tree is ' $S_{0} . S_{2}$ 'shown in fig. 6.

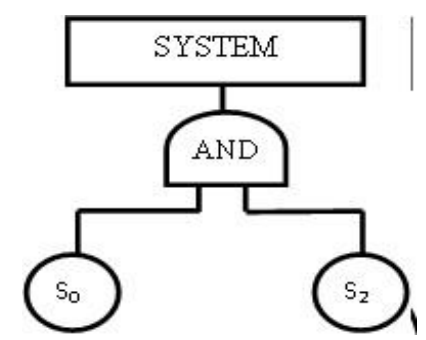

Fig.6. Fault tree when relay node fails

$$
R=P Y
$$

RELIABILITY $=1-P Y$

Case 3: Network State when member node has failed

The fault tree denotes the member node failure case and computes the reliability as depicted in equation (13) and (14). The system fails if $\{P Q Y\}$ is 1 or when the output of the corresponding fault tree is ' $S_{0} . S_{3} \cdot S_{2}$ ', as shown in fig. 7.

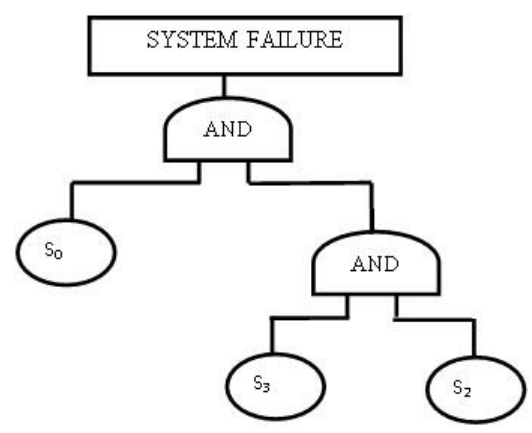

Fig.7. Fault tree when member node fails 


$$
R=P(Q Y)
$$

$$
\text { RELIABILITY }=1-P Q Y
$$

\section{Conclusions}

A wireless sensor network is characterised by energy constricts, intermittent mobility, localization problems. For the simplicity of analysis, we assume a sensor network that has a fixed topology based message transmission system. Among these networks, one of the most popular technologies is context to wireless sensor networks is based on IEEE standards 802.11s and 802.15.4 (Zigbee network).

The article aims to depict the fault tree models for mainly two types of network that are used in the Zigbee based Wireless Sensor Networks. Different failure cases that can occur during message transfer in a network, such as a link failure, relay node failure, etc are elaborately analysed and modelled through the fault tree model. The article outlines and analyses the effect on the reliability of the network in case the network elements fail. It also tries to present a detailed analysis of the effect of the failure of network elements on the overall communication network structure.

The reliability estimates can be further used to propose strategies to prolonging lifetime of the critical elements that affect the overall health of the network. The reliability estimates can thus be applied to different networks to estimate the reliability of any type of network.

\section{References}

[1] Lee, Myung J., Jianliang Zheng, Young-Bae Ko, and Deepesh Man Shrestha. "Emerging standards for wireless mesh technology." Wireless Communications, IEEE 13, no. 2 (2006): 56-63.

[2] Snigdh, Itu, and Nisha Gupta. "Enhancing Reliability in Backbone Assisted Wireless Sensor Networks." International Journal of Performability Engineering 11.5 (2015).

[3] Arfat, Yasir, and Riaz Ahmed Shaikh. "A Survey on Secure Routing Protocols in Wireless Sensor Networks." (2016).

[4] Hiertz, Guido R., Dee Denteneer, Philips LotharStibor, YunpengZang, Xavier Pérez Costa, and Bernhard Walke. "The IEEE 802.11 universe. "Communications Magazine, IEEE 48, no. 1 (2010): 62-70.

[5] Malhotra, Jyoteesh. "On the Selection of Physical Layer Optimized Network Topologies for the Zigbee Network." International Journal of Information Technology and Computer Science (IJITCS) 8.1 (2016): 84.

[6] Kinney, Patrick. "Zigbee technology: Wireless control that simply works. "Communications design conference. Vol.2. 2003.

[7] Kusiak, Andrew. Engineering design: products, processes, and systems. Academic Press, Inc., 1999.

[8] Kermarrec, Anne-Marie, Laurent Massoulié, and Ayalvadi J. Ganesh. "Probabilistic reliable dissemination in large-scale systems." Parallel and Distributed Systems, IEEE Transactions on 14.3 (2003): 248-258.

[9] Biradar, Rajashekhar, SunilkumarManvi, and Mylara Reddy. "Link stability based multicast routing scheme in MANET." Computer Networks 54.7 (2010): 1183-1196.

[10] Chen, Shyr-Kuen, Tsair Kao, Chia-Tai Chan, Chih-Ning Huang, Chih-Yen Chiang, Chin-Yu Lai, Tse-Hua Tung, and Pi-Chung Wang. "A reliable transmission protocol for ZigBee-based wireless patient monitoring. "Information Technology in Biomedicine, IEEE Transactions on 16, no. 1 (2012): 6-16.

[11] Kumar, Vijay, R. B. Patel, Manpreet Singh, and RohitVaid. "Markov Model for Reliable Packet Delivery in Wireless Sensor Networks." International Journal of Computer Science Issues 8, no. 3 (2011): 16940814.

[12] Park, Seung-Jong, RamanujaVedantham, RaghupathySivakumar, and Ian F. Akyildiz. "A scalable 
approach for reliable downstream data delivery in wireless sensor networks."In Proceedings of the 5th ACM international symposium on Mobile ad hoc networking and computing, pp. 78-89.ACM, 2004.

[13] Wang, Chaonan, Liudong Xing Vinod M. Vokkarane, and Yan Sun. "Reliability of wireless sensor networks with tree topology. " International Journal of Performability Engineering 8.2 (2012): 213.

[14] Chaturvedi, Sanjay K., and N. Padmavathy. "The Influence of Scenario Metrics on Network Reliability of Mobile Ad Hoc Network."International Journal of Performability Engineering 9.1 (2013).

[15] Peng, Rui, QingqingZhai, Liudong Xing, and Jun Yang. "Reliability of 1-out-of-(n+ 1) Warm Standby Systems Subject to Fault Level Coverage."International Journal of Performability Engineering 9, no.1 (2013).

[16] Shrestha, Akhilesh, Liudong Xing, Yan Sun, and Vinod M. Vokkarane. "Infrastructure Communication Reliability of Wireless Sensor Networks Considering Common-Cause Failures." International Journal of Performability Engineering 8, no. 2 (2012).

[17] Silva, Ivanovitch, Luiz AffonsoGuedes, Paulo Portugal, and Francisco Vasques. "Reliability and availability evaluation of wireless sensor networks for industrial applications." Sensors 12, no. 1 (2012): 806-838.

[18] Dâmaso, Antônio, Nelson Rosa, and Paulo Maciel. "Reliability of wireless sensor networks. "Sensors 14.9 (2014): 15760-15785.

\section{Authors' Profiles}

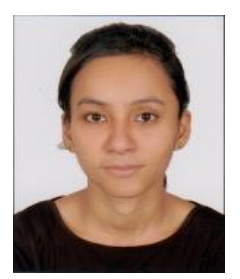

Apoorva Singh is currently pursuing M.Tech. degree in Computer Science from Birla Institute of Technology, Mesra, Ranchi, India. She received her B.Tech degree in Computer Science and Engineering from Rajiv Gandhi Technical University, Bhopal, India, in 2014. Her research interests include WSN networks and mobile ad hoc networks.

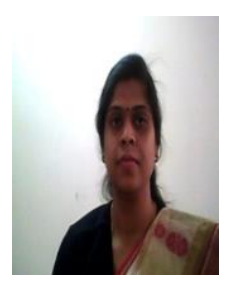

Itu Snigdh completed her Ph.D in 2016 in the research area of wireless sensor networks and her Masters Degree (Software Engineering) from B.I.T Mesra (Ranchi), India in 2002. Her Bachelor's degree is in Electrical Engineering from B.I.T Sindri, India in 1996. She joined the Department of Computer Science and Engineering at BIT Mesra in 2003 and is currently working as an Assistant Professor. She has authored and coauthored a number of technical journal articles and conference papers. Her areas of interest include Software Engineering, Database Management Systems, Mobile Computing and Wireless Sensor Networks.

How to cite this paper: Apoorva Singh, Itu Snigdh,"Modelling Failure Conditions in Zigbee based Wireless Sensor Networks", International Journal of Wireless and Microwave Technologies(IJWMT), Vol.7, No.2, pp.25-34, 2017.DOI: 10.5815/ijwmt.2017.02.03 